CRYSTALLOGRAPHIC COMMUNICATIONS

ISSN 2056-9890

Received 12 April 2018

Accepted 24 May 2018

Edited by K. Fejfarova, Institute of Biotechnology CAS, Czech Republic

Keywords: azopyrrole; crystal structure; hydrogen bonding.

CCDC reference: 1845021

Supporting information: this article has supporting information at journals.iucr.org/e

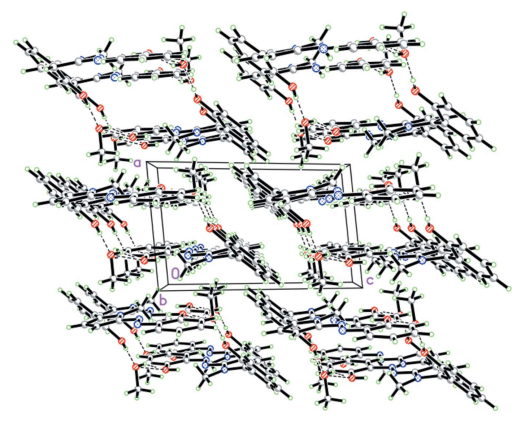

OPEN $\odot$ ACCESS

\section{Crystal structure of 2-\{5-[2-(2-hydroxyphenyl)- diazen-1-yl]-1-methylpyrrol-2-yl\}phenol methanol monosolvate}

\author{
Guiwen Yang, Huixiao Feng and Zhenming Yin*
}

College of Chemistry, Tianjin Key Laboratory of Structure and Performance for, Functional Molecule, Tianjin Normal University, Tianjin 300387, People's Republic of China;, Key Laboratory of Inorganic-Organic Hybrid Functional Materials Chemistry, (Tianjin Normal University), Ministry of Education, Tianjin 300387, People's Republic of China. *Correspondence e-mail: tjyinzm@aliyun.com

In the title azopyrrole compound, $\mathrm{C}_{17} \mathrm{H}_{15} \mathrm{~N}_{3} \mathrm{O}_{2} \cdot \mathrm{CH}_{3} \mathrm{OH}$, the azo $\mathrm{N}=\mathrm{N}$ bond adopts a trans configuration and the pyrrole $\mathrm{N}$ and azo group are in an anti orientation. The dihedral angles between the pyrrole ring and the two phenyl rings are $6.7(3)$ and $54.7(3)^{\circ}$. In the crystal, a supramolecular ring structure is formed between two azopyrrole and two methanol solvent molecules through four $\mathrm{O}-\mathrm{H}$... O hydrogen bonds.

\section{Chemical context}

Recently, azopyrrole dyes have received much attention for their promising use in the design of advanced materials and devices. For example, some thienylpyrrole azo dyes bearing heterocyclic groups have good non-linear optical properties (Raposo et al., 2011). Mikroyannidis and coworkers found that many azopyrrole dyes are efficient bulk heterojunction solar cell materials (Sharma et al., 2012). In a previous work, we reported the crystal engineering of some 5,5'-bis(phenyldiazo)dipyrromethane compounds and demonstrated their interlocked type self-assemblies in the solid state via quadruple $\mathrm{N}-\mathrm{H} \cdots \mathrm{N}$ hydrogen bonds (Yin et al., 2008, 2009). In a continuation of this research, we report herein the crystal structure of 2-\{5-[2-(2-hydroxyphenyl)diazen-1-yl]-1-methylpyrrol-2-yl\}phenol methanol monosolvate.<smiles>COc1ccccc1/N=N/c1ccc(-c2ccccc2O)n1C</smiles>

\section{Structural commentary}

The structure of the title compound is shown in Fig. 1. The asymmetric unit contains one azopyrrole molecule and one methanol solvent molecule. The azoylazopyrrole group is almost planar, reflected by the dihedral angle between the pyrrole ring $(\mathrm{N} 3 / \mathrm{C} 7-\mathrm{C} 10)$ and the benzene ring $(\mathrm{C} 1-\mathrm{C} 6)$ of only $6.7(3)^{\circ}$, which may be due to the existence of the intra- 


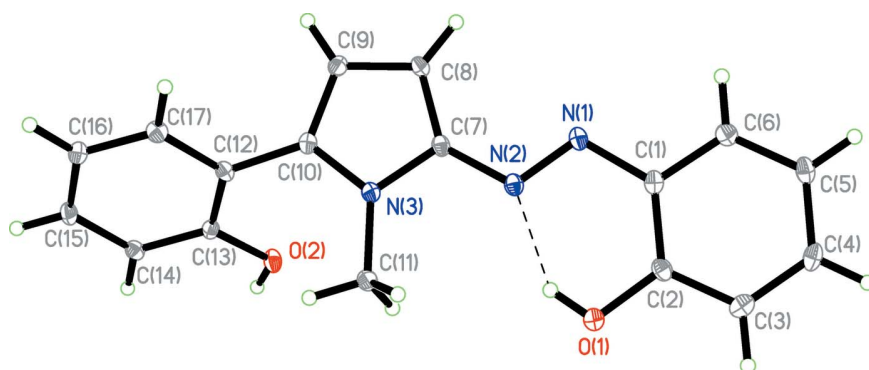

Figure 1

ORTEP diagram for the title compound, with displacement ellipsoids drawn at the $30 \%$ probability level. The methanol solvent molecule was omitted for clarity.

molecular $\mathrm{O} 1-\mathrm{H} 1 \cdots \mathrm{N} 2$ hydrogen bond (Table 1) between the hydroxy group and the azo $\mathrm{N}$ atom. The dihedral angle between pyrrole ring and the other benzene ring $(\mathrm{C} 12-\mathrm{C} 17)$ is $54.7(3)^{\circ}$, which may be caused by the steric repulsion between hydroxy group and methyl group. The azo $\mathrm{N}=\mathrm{N}$ bond adopts a trans configuration and its length is $1.286(2) \AA$, which is shorter than that in the crystal of 2,5-bis(2-hydroxyphenylazo)- $1 H$-pyrrole (1.293 $\AA$; Li et al. 2009). It is worth mentioning that the $\mathrm{N} 1$ atom of the azo group and the N3 atom of the pyrrole ring are arranged on opposite sides with respect to the $\mathrm{C} 7-\mathrm{N} 2$ bond, which is the same as in the crystal of 2-phenylazo-1-vinyl pyrrole (Trofimov et al., 2006) but different to many other observations (Li et al., 2009; Yin et al., 2008). The bond lengths in the pyrrole ring are more equal compared to those normally observed.

\section{Supramolecular features}

In the crystal, two azopyrrole molecules are bridged by two methanol solvent molecules through four $\mathrm{O}-\mathrm{H} \cdots \mathrm{O}$

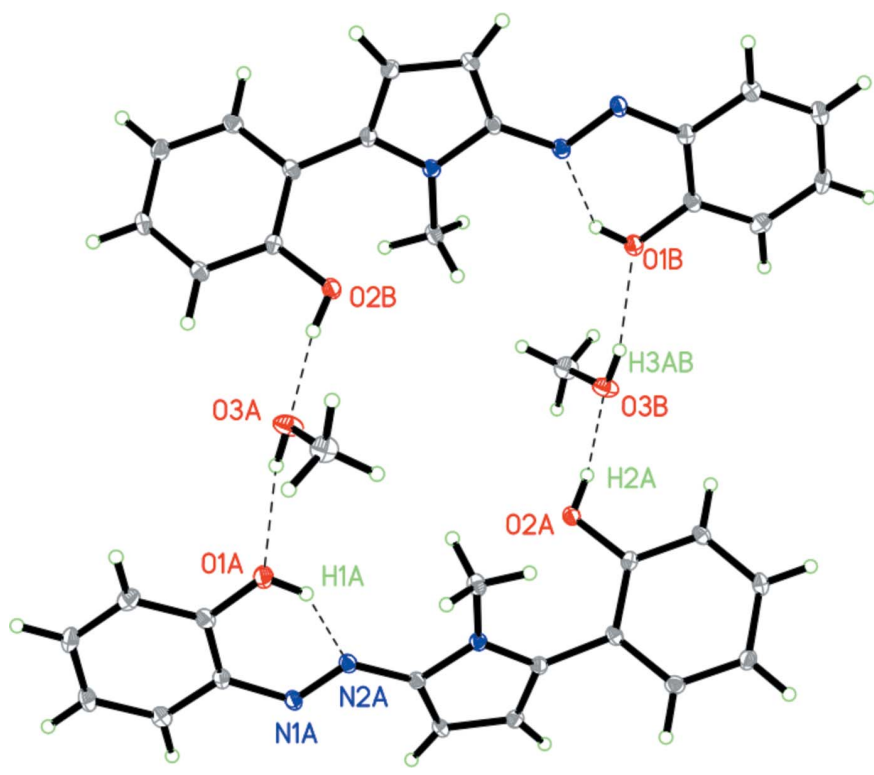

Figure 2

$\mathrm{O}-\mathrm{H} \cdots \mathrm{O}$ hydrogen-bonded (Table 1) supramolecular ring structure.
Table 1

Hydrogen-bond geometry $\left(\AA,^{\circ}\right)$.

$C g 1$ and $C g 2$ are the centroids of the $\mathrm{C} 1-\mathrm{C} 6$ and $\mathrm{C} 12-\mathrm{C} 17$ rings, respectively

\begin{tabular}{lllll}
\hline$D-\mathrm{H} \cdots A$ & $D-\mathrm{H}$ & $\mathrm{H} \cdots A$ & $D \cdots A$ & $D-\mathrm{H} \cdots A$ \\
\hline $\mathrm{O} 1-\mathrm{H} 1 \cdots \mathrm{N} 2$ & 0.84 & 1.81 & $2.530(2)$ & 143 \\
$\mathrm{O} 2-\mathrm{H} 2 \cdots 3^{\mathrm{i}}$ & 0.84 & 1.81 & $2.641(2)$ & 171 \\
$\mathrm{O} 3-\mathrm{H} 3 A \cdots \mathrm{O} 1^{\mathrm{ii}}$ & 0.84 & 1.97 & $2.763(2)$ & 157 \\
$\mathrm{C} 11-\mathrm{H} 11 B \cdots C g 1^{\mathrm{iii}}$ & 0.98 & 2.73 & $3.587(2)$ & 147 \\
$\mathrm{C} 18-\mathrm{H} 18 C \cdots C g 2^{\text {iv }}$ & 0.98 & 2.75 & $3.483(3)$ & 132 \\
\hline
\end{tabular}

Symmetry codes: (i) $-x+1,-y+2,-z+1$; (ii) $x, y, z+1$; (iii) $-x,-y+1,-z$; (iv) $-x,-y+2,-z+1$.

hydrogen bonds forming a large supramolecular ring structure, in which the methanol acts as both a hydrogen-bond acceptor and a donor (Fig. 2, Table 1). This type of coordination environment is most populated (occupying 70\%) for methanol molecules as revealed by a search of the Cambridge Structural Database (CSD) (Brychczynska et al. 2008). The methyl groups point to the inside of the ring. The rings are further held together through $\mathrm{C}-\mathrm{H} \cdots \pi$ contacts involving the benzene rings (Table 1 ). There are no $\pi-\pi$ interactions between the aromatic rings. The packing is shown in Fig. 3.

\section{Database survey}

A search in the Cambridge Structural Database (Version 5.38; Groom et al., 2016) returned 45 entries for azopyrrole derivatives, including three entries for $N$-vinylphenylazopyrrole (Trofimov et al., 2006; Rusakov et al., 2007), four entries for mono- or bisazopyrroles (Li et al., 2009), two entries for azo calix[4]pyrroles (Nishiyabu et al., 2006), five entries for pyrrole-azocrown ethers (Wagner-Wysiecka et al., 2011; Szczygelska-Tao et al., 2008), two entries for azopyrrole boron difluoride complexes ( $\mathrm{Li}$ et al., 2009; Lee et al., 2012), ten entries for metal complexes (Li et al., 2008; Li, \& Dolphin, 2011; Yin et al., 2012; Zhang et al., 2015; Ghorui et al., 2016);

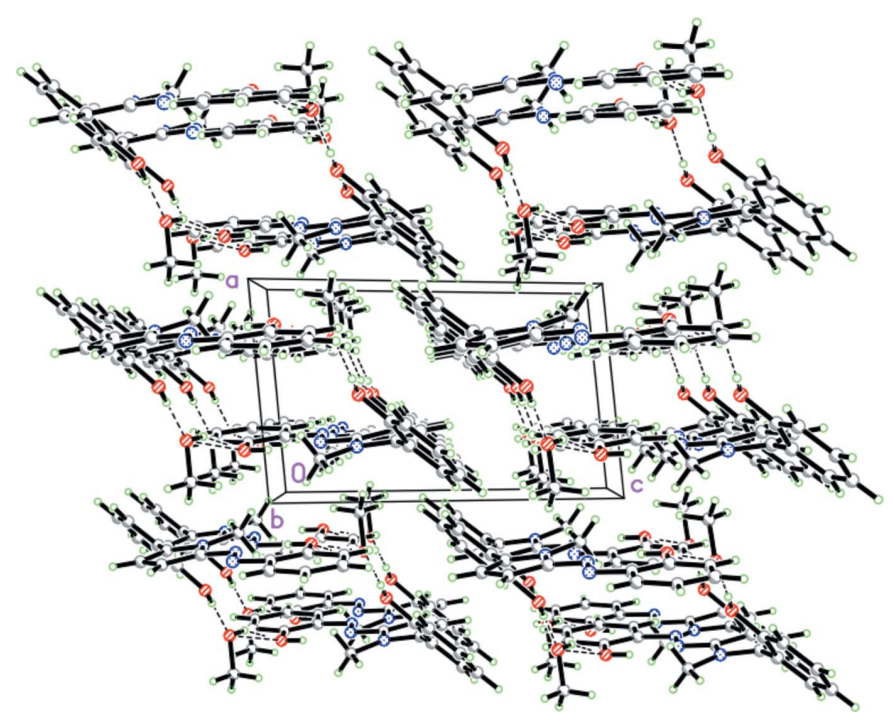

Figure 3

A view of the crystal packing along the $b$ axis. 
the majority are for mono- or bisphenylazodipyrromethanes (Yin et al., 2008, 2009; Chen \& Yin, 2014; Zhang \& Yin, 2014).

\section{Synthesis and crystallization}

A $273 \mathrm{~K}$ solution of 2-aminophenol $0.272 \mathrm{~g}(2.5 \mathrm{mmol})$ and aqueous $\mathrm{HCl}(2 \mathrm{~mL})$ in water $(2 \mathrm{~mL})$ was treated with another $273 \mathrm{~K}$ solution of $\mathrm{NaNO}_{2}(0.18 \mathrm{~g}, 2.5 \mathrm{mmol})$ in $3 \mathrm{~mL}$ water, and the mixture was stirred at $273 \mathrm{~K}$ for $30 \mathrm{~min}$. The diazonium salt solution was added dropwise to a solution of $\mathrm{N}$ methylpyrrole $(81 \mathrm{mg}, 1 \mathrm{mmol})$ in acetonitrile $(25 \mathrm{~mL})$ and three drops of acetic acid. The combined solution was maintained at $273 \mathrm{~K}$ for $2 \mathrm{~h}$ with stirring. After that, EtOAc $(25 \mathrm{~mL})$ and water $(25 \mathrm{~mL})$ were added. The organic layer was separated and washed with water $(20 \mathrm{~mL})$ and dried with anhydrous $\mathrm{MgSO}_{4}$. The solution was evaporated and the residue was purified by column chromatography on silica (ethyl acetate/petroleum ether $=1: 2$ ), which gave the title compound as an orange powder ( $200 \mathrm{mg}, 68 \%$, m.p. $404 \mathrm{~K})$.

${ }^{1} \mathrm{H}$ NMR $\left(400 \mathrm{MHz}\right.$, DMSO- $\left.d_{6}\right): \delta 3.73\left(s, 3 \mathrm{H},-\mathrm{CH}_{3}\right), 6.32$ $(d, J=4 \mathrm{~Hz}, 1 \mathrm{H}$, pyrrole $\mathrm{C}-\mathrm{H}), 6.86(d, J=4 \mathrm{~Hz}, 1 \mathrm{H}$, pyrrole C-H), 6.90-6.95 (m, 2H, Ar C-H), $6.99(t, 2 \mathrm{H}, J=8 \mathrm{~Hz}, \mathrm{Ar} \mathrm{C}-$ $\mathrm{H}), 7.23-7.31(m, 3 \mathrm{H}, \mathrm{Ar} \mathrm{C}-\mathrm{H}), 7.65(d, J=8 \mathrm{~Hz}, 1 \mathrm{H}, \mathrm{Ar} \mathrm{C}-$ $\mathrm{H}), 9.95(s, 1 \mathrm{H},-\mathrm{OH}), 10.43(s, 1 \mathrm{H},-\mathrm{OH})$. Crystals suitable $\mathrm{X}$-ray diffraction analysis were obtained by the slow evaporation of a $\mathrm{CHCl}_{3} / \mathrm{CH}_{3} \mathrm{OH}$ solution of the title compound.

\section{Refinement}

Crystal data, data collection and structure refinement details are summarized in Table 2. $\mathrm{O}-\mathrm{H}$ atoms were located in a difference-Fourier map and refined freely. Other $\mathrm{H}$ atoms were positioned geometrically $(\mathrm{C}-\mathrm{H}=0.95$ or $0.98 \AA$ ) and included in the final cycles of refinement using a riding model, with $U_{\text {iso }}(\mathrm{H})=1.2 U_{\text {eq }}(\mathrm{C})$ or $1.5 U_{\text {eq }}($ Cmethyl $)$.

\section{Funding information}

Funding for this research was provided by: National Natural Science Foundation of China (award No. 21172174).

\section{References}

Bruker (2005). APEX2, SAINT and SADABS. Bruker AXS Inc., Madison, Wisconsin, USA.

Brychczynska, M., Davey, R. J. \& Pidcock, E. (2008). New J. Chem. 32, 1754-1760.

Chen, J. \& Yin, Z. (2014). Dyes Pigments, 102, 94-99.

Ghorui, T., Roy, S., Pramanik, S. \& Pramanik, K. (2016). Dalton Trans. 45, 5720-5729.

Groom, C. R., Bruno, I. J., Lightfoot, M. P. \& Ward, S. C. (2016). Acta Cryst. B72, 171-179.

Lee, H. Y., Olasz, A., Chen, C.-H. \& Lee, D. (2012). Org. Lett. 14, 6286-6289.

Li, Y. \& Dolphin, D. (2011). Can. J. Chem. 89, 481-487.

Li, W.-Y., Mao, L.-S., Lu, L. \& He, H.-W. (2008). Acta Cryst. E64, $\mathrm{m} 490$.
Table 2

Experimental details.

\begin{tabular}{|c|c|}
\hline \multicolumn{2}{|l|}{ Crystal data } \\
\hline Chemical formula & $\mathrm{C}_{17} \mathrm{H}_{15} \mathrm{~N}_{3} \mathrm{O}_{2} \cdot \mathrm{CH}_{4} \mathrm{O}$ \\
\hline$M_{\mathrm{r}}$ & 325.36 \\
\hline Crystal system, space group & Triclinic, $P \overline{1}$ \\
\hline Temperature (K) & 173 \\
\hline$a, b, c(\AA)$ & $7.1597(11), 9.8762(16), 12.243(2)$ \\
\hline$\alpha, \beta, \gamma\left({ }^{\circ}\right)$ & $110.426(3), 94.051(3), 93.378$ (3) \\
\hline$V\left(\AA^{3}\right)$ & $806.0(2)$ \\
\hline$Z$ & 2 \\
\hline Radiation type & Мо $K \alpha$ \\
\hline$\mu\left(\mathrm{mm}^{-1}\right)$ & 0.09 \\
\hline Crystal size $(\mathrm{mm})$ & $0.14 \times 0.13 \times 0.12$ \\
\hline \multicolumn{2}{|l|}{ Data collection } \\
\hline Diffractometer & Bruker APEXII CCD \\
\hline Absorption correction & $\begin{array}{l}\text { Multi-scan (SADABS; Bruker, } \\
\text { 2005) }\end{array}$ \\
\hline$T_{\min }, T_{\max }$ & $0.987,0.989$ \\
\hline $\begin{array}{l}\text { No. of measured, independent and } \\
\text { observed }[I>2 \sigma(I)] \text { reflections }\end{array}$ & $4168,2830,2071$ \\
\hline$R_{\text {int }}$ & 0.025 \\
\hline$(\sin \theta / \lambda)_{\max }\left(\AA^{-1}\right)$ & 0.595 \\
\hline \multicolumn{2}{|l|}{ Refinement } \\
\hline$R\left[F^{2}>2 \sigma\left(F^{2}\right)\right], w R\left(F^{2}\right), S$ & $0.045,0.109,1.03$ \\
\hline No. of reflections & 2830 \\
\hline No. of parameters & 222 \\
\hline $\mathrm{H}$-atom treatment & H-atom parameters constrained \\
\hline$\Delta \rho_{\max }, \Delta \rho_{\min }\left(\mathrm{e} \AA^{-3}\right)$ & $0.21,-0.23$ \\
\hline
\end{tabular}

Computer programs: APEX2 and SAINT (Bruker, 2005), SHELXS97, SHELXL97 and SHELXTL (Sheldrick, 2008).

Li, Y., Patrick, B. O. \& Dolphin, D. (2009). J. Org. Chem. 74, 52375243.

Nishiyabu, R., Palacios, M. A., Dehaen, W. \& Anzenbacher, P. Jr (2006). J. Am. Chem. Soc. 128, 11496-11504.

Raposo, M. M. M. M., Castro, C. R., Fonseca, A. M. C., Schellenberg, P. \& Belsley, M. (2011). Tetrahedron, 67, 5189-5198.

Rusakov, Y. Y., Krivdin, L. B., Senotrusova, E. Y., Schmidt, E. Y., Vasiltsov, A. M., Mikhaleva, A. I., Trofimov, B. A., Dyachenko, O. A., Chekhlov, A. N. \& Kazheva, O. N. (2007). Magn. Reson. Chem. 45, 142-151.

Sharma, G. D., Mikroyannidis, J. A., Sharma, S. S. \& Justin Thomas, K. R. (2012). Dyes Pigments, 94, 320-329.

Sheldrick, G. M. (2008). Acta Cryst. A64, 112-122.

Szczygelska-Tao, J., Fonari, M. S. \& Biernat, J. F. (2008). Supramol. Chem. 20, 651-658.

Trofimov, B. A., Schmidt, Y. E., Mikhaleva, A. I., Vasil'tsov, A. M., Zaitsev, A. B., Smolyanina, N. S., Senotrusova, E. Y., Afonin, A. V., Ushakov, I. A., Petrushenko, K. B., Kazheva, O. N., Dyachenko, O. A., Smirnov, V. V., Schmidt, A. F., Markova, M. V. \& Morozova, L. V. (2006). Eur. J. Org. Chem. pp. 4021-4033.

Wagner-Wysiecka, E., Rzymowski, T., Fonari, M. S., Kulmaczewski, R. \& Luboch, E. (2011). Tetrahedron, 67, 1862-1872.

Yin, Z., Wang, W., Du, M., Wang, X. \& Guo, J. (2009). CrystEngComm, 11, 2441-2446.

Yin, Z., Wang, W., Guo, J., Wang, J., He, J. \& Cheng, J.-P. (2008). CrystEngComm, 10, 957-959.

Yin, Z., Yan, Y., Sun, S. \& Wang, W. (2012). J. Coord. Chem. 65, 865874.

Zhang, H., Chen, J. \& Yin, Z. (2015). Chin. J. Struct. Chem. 34, 18761882.

Zhang, H. \& Yin, Z. (2014). Chin. J. Struct. Chem. 33, 1813-1818. 


\section{supporting information}

Acta Cryst. (2018). E74, 871-873 [https://doi.org/10.1107/S2056989018007764]

Crystal structure of 2-\{5-[2-(2-hydroxyphenyl)diazen-1-yl]-1-methylpyrrol-2yl\}phenol methanol monosolvate

\section{Guiwen Yang, Huixiao Feng and Zhenming Yin}

Computing details

Data collection: APEX2 (Bruker, 2005); cell refinement: SAINT (Bruker, 2005); data reduction: SAINT (Bruker, 2005); program(s) used to solve structure: SHELXS97 (Sheldrick, 2008); program(s) used to refine structure: SHELXL97

(Sheldrick, 2008); molecular graphics: SHELXTL (Sheldrick, 2008); software used to prepare material for publication: SHELXTL (Sheldrick, 2008).

2-\{5-[2-(2-Hydroxyphenyl)diazen-1-yl]-1-methylpyrrol-2-yl\}phenol methanol monosolvate

\section{Crystal data}

$\mathrm{C}_{17} \mathrm{H}_{15} \mathrm{~N}_{3} \mathrm{O}_{2} \cdot \mathrm{CH}_{4} \mathrm{O}$

$M_{r}=325.36$

Triclinic, $P \overline{1}$

$a=7.1597(11) \AA$

$b=9.8762(16) \AA$

$c=12.243(2) \AA$

$\alpha=110.426(3)^{\circ}$

$\beta=94.051(3)^{\circ}$

$\gamma=93.378(3)^{\circ}$

$V=806.0(2) \AA^{3}$

\section{Data collection}

Bruker APEXII CCD

diffractometer

Radiation source: fine-focus sealed tube

Graphite monochromator

$\varphi$ and $\omega$ scans

Absorption correction: multi-scan

(SADABS; Bruker, 2005)

$T_{\min }=0.987, T_{\max }=0.989$

Refinement

Refinement on $F^{2}$

Least-squares matrix: full

$R\left[F^{2}>2 \sigma\left(F^{2}\right)\right]=0.045$

$w R\left(F^{2}\right)=0.109$

$S=1.03$

2830 reflections

222 parameters

0 restraints
$Z=2$

$F(000)=344$

$D_{\mathrm{x}}=1.341 \mathrm{Mg} \mathrm{m}^{-3}$

Mo $K \alpha$ radiation, $\lambda=0.71073 \AA$

Cell parameters from 1104 reflections

$\theta=2.3-28.1^{\circ}$

$\mu=0.09 \mathrm{~mm}^{-1}$

$T=173 \mathrm{~K}$

BLOCK, yellow

$0.14 \times 0.13 \times 0.12 \mathrm{~mm}$

4168 measured reflections

2830 independent reflections

2071 reflections with $I>2 \sigma(I)$

$R_{\text {int }}=0.025$

$\theta_{\text {max }}=25.0^{\circ}, \theta_{\min }=1.8^{\circ}$

$h=-8 \rightarrow 8$

$k=-11 \rightarrow 9$

$l=-14 \rightarrow 14$

Primary atom site location: structure-invariant direct methods

Secondary atom site location: difference Fourier map

Hydrogen site location: inferred from neighbouring sites

$\mathrm{H}$-atom parameters constrained 
$w=1 /\left[\sigma^{2}\left(F_{\mathrm{o}}^{2}\right)+(0.0437 P)^{2}+0.2373 P\right]$

where $P=\left(F_{\mathrm{o}}^{2}+2 F_{\mathrm{c}}{ }^{2}\right) / 3$

$(\Delta / \sigma)_{\max }<0.001$

$$
\Delta \rho_{\max }=0.21 \mathrm{e} \AA^{-3}
$$

$\Delta \rho_{\min }=-0.23$ e $\AA^{-3}$

\section{Special details}

Geometry. All esds (except the esd in the dihedral angle between two 1.s. planes) are estimated using the full covariance matrix. The cell esds are taken into account individually in the estimation of esds in distances, angles and torsion angles; correlations between esds in cell parameters are only used when they are defined by crystal symmetry. An approximate (isotropic) treatment of cell esds is used for estimating esds involving 1.s. planes.

Refinement. Refinement of $\mathrm{F}^{2}$ against ALL reflections. The weighted $\mathrm{R}$-factor $\mathrm{wR}$ and goodness of fit $\mathrm{S}$ are based on $\mathrm{F}^{2}$, conventional R-factors $R$ are based on $F$, with $F$ set to zero for negative $F^{2}$. The threshold expression of $F^{2}>2$ sigma $\left(F^{2}\right)$ is used only for calculating R-factors(gt) etc. and is not relevant to the choice of reflections for refinement. R-factors based on $\mathrm{F}^{2}$ are statistically about twice as large as those based on F, and R-factors based on ALL data will be even larger.

Fractional atomic coordinates and isotropic or equivalent isotropic displacement parameters $\left(\AA^{2}\right)$

\begin{tabular}{|c|c|c|c|c|}
\hline & $x$ & $y$ & $z$ & $U_{\text {iso }} * / U_{\text {eq }}$ \\
\hline $\mathrm{O} 1$ & $0.2218(2)$ & $0.53355(15)$ & $-0.07978(12)$ & $0.0254(4)$ \\
\hline H1 & 0.2260 & 0.5941 & -0.0113 & $0.038 *$ \\
\hline $\mathrm{O} 2$ & $0.4830(2)$ & $1.13606(14)$ & $0.27561(13)$ & $0.0221(4)$ \\
\hline $\mathrm{H} 2$ & 0.5527 & 1.1998 & 0.2636 & $0.033 *$ \\
\hline $\mathrm{O} 3$ & 0.2725 & $0.68674(17)$ & $0.77244(15)$ & $0.0304(4)$ \\
\hline $\mathrm{H} 3 \mathrm{~A}$ & 0.2900 & 0.6375 & 0.8154 & $0.046^{*}$ \\
\hline N1 & $0.2920(2)$ & $0.48800(18)$ & $0.14369(15)$ & $0.0191(4)$ \\
\hline $\mathrm{N} 2$ & $0.2614(2)$ & $0.61395(18)$ & $0.14132(14)$ & $0.0181(4)$ \\
\hline N3 & $0.2453(2)$ & $0.86125(17)$ & $0.24967(14)$ & $0.0166(4)$ \\
\hline $\mathrm{C} 1$ & 0.2840 & $0.3796(2)$ & $0.03169(18)$ & $0.0180(5)$ \\
\hline $\mathrm{C} 2$ & 0.2539 & $0.4013(2)$ & $-0.07572(18)$ & $0.0196(5)$ \\
\hline $\mathrm{C} 3$ & $0.2592(3)$ & $0.2857(2)$ & -0.18007 (19) & $0.0247(5)$ \\
\hline $\mathrm{H} 3$ & 0.2390 & 0.3001 & -0.2526 & $0.030 *$ \\
\hline $\mathrm{C} 4$ & $0.2933(3)$ & $0.1506(2)$ & -0.17897 (19) & $0.0243(5)$ \\
\hline $\mathrm{H} 4$ & 0.2988 & 0.0728 & -0.2508 & $0.029 *$ \\
\hline $\mathrm{C} 5$ & $0.3199(3)$ & $0.1269(2)$ & $-0.07380(19)$ & $0.0235(5)$ \\
\hline H5 & 0.3407 & 0.0329 & -0.0735 & $0.028 *$ \\
\hline C6 & $0.3159(3)$ & $0.2406(2)$ & 0.02965 (19) & $0.0206(5)$ \\
\hline H6 & 0.3353 & 0.2244 & 0.1015 & $0.025^{*}$ \\
\hline $\mathrm{C} 7$ & $0.2752(3)$ & $0.7239(2)$ & $0.24820(17)$ & $0.0170(5)$ \\
\hline $\mathrm{C} 8$ & $0.3197(3)$ & $0.7316(2)$ & $0.36227(17)$ & $0.0190(5)$ \\
\hline $\mathrm{H} 8$ & 0.3478 & 0.6533 & 0.3870 & $0.023^{*}$ \\
\hline C9 & $0.3152(3)$ & $0.8753(2)$ & $0.43346(18)$ & $0.0190(5)$ \\
\hline H9 & 0.3386 & 0.9128 & 0.5164 & $0.023^{*}$ \\
\hline $\mathrm{C} 10$ & 0.2708 & $0.9552(2)$ & $0.36319(17)$ & $0.0172(5)$ \\
\hline $\mathrm{C} 11$ & 0.1888 & $0.8954(2)$ & $0.14600(18)$ & $0.0210(5)$ \\
\hline H11A & 0.1466 & 0.9933 & 0.1702 & $0.032 *$ \\
\hline H11B & 0.0858 & 0.8251 & 0.0986 & $0.032 *$ \\
\hline $\mathrm{H} 11 \mathrm{C}$ & 0.2959 & 0.8908 & 0.0997 & $0.032 *$ \\
\hline $\mathrm{C} 12$ & 0.2552 & $1.1127(2)$ & $0.39959(17)$ & $0.0167(5)$ \\
\hline $\mathrm{C} 13$ & 0.3645 & $1.2011(2)$ & $0.35662(17)$ & $0.0166(5)$ \\
\hline $\mathrm{C} 14$ & 0.3540 & $1.3501(2)$ & $0.39739(17)$ & $0.0184(5)$ \\
\hline
\end{tabular}




\begin{tabular}{lllll} 
H14 & 0.4266 & 1.4092 & 0.3664 & $0.022^{*}$ \\
C15 & $0.2372(3)$ & $1.4124(2)$ & $0.48346(18)$ & $0.0203(5)$ \\
H15 & 0.2299 & 1.5144 & 0.5113 & $0.024^{*}$ \\
C16 & $0.1314(3)$ & $1.3269(2)$ & $0.52895(18)$ & $0.0211(5)$ \\
H16 & 0.0526 & 1.3699 & 0.5886 & $0.025^{*}$ \\
C17 & $0.1411(3)$ & $1.1790(2)$ & $0.48707(18)$ & $0.0201(5)$ \\
H17 & 0.0682 & 1.1207 & 0.5187 & $0.024^{*}$ \\
C18 & $0.0860(3)$ & $0.7274(3)$ & $0.7761(2)$ & $0.0310(6)$ \\
H18A & 0.0712 & 0.7970 & 0.8541 & $0.046^{*}$ \\
H18B & -0.0020 & 0.6411 & 0.7594 & $0.046^{*}$ \\
H18C & 0.0596 & 0.7720 & 0.7173 & $0.046^{*}$ \\
\hline
\end{tabular}

Atomic displacement parameters $\left(\AA^{2}\right)$

\begin{tabular}{lllllll}
\hline & $U^{11}$ & $U^{22}$ & $U^{33}$ & $U^{12}$ & $U^{13}$ & $U^{23}$ \\
\hline O1 & $0.0358(9)$ & $0.0192(8)$ & $0.0215(8)$ & $0.0050(7)$ & $0.0042(7)$ & $0.0068(7)$ \\
O2 & $0.0216(8)$ & $0.0161(8)$ & $0.0286(8)$ & $0.0020(6)$ & $0.0093(7)$ & $0.0066(7)$ \\
O3 & $0.0220(8)$ & $0.0362(10)$ & $0.0448(10)$ & $0.0055(7)$ & $0.0064(7)$ & $0.0279(8)$ \\
N1 & $0.0188(9)$ & $0.0144(9)$ & $0.0233(10)$ & $0.0014(7)$ & $0.0030(8)$ & $0.0053(8)$ \\
N2 & $0.0164(9)$ & $0.0155(10)$ & $0.0216(9)$ & $0.0004(7)$ & $0.0024(7)$ & $0.0057(8)$ \\
N3 & $0.0157(9)$ & $0.0155(9)$ & $0.0188(9)$ & $0.0018(7)$ & $0.0022(7)$ & $0.0062(7)$ \\
C1 & $0.0120(10)$ & $0.0167(11)$ & $0.0226(11)$ & $0.0005(8)$ & $0.0008(9)$ & $0.0040(9)$ \\
C2 & $0.0165(11)$ & $0.0176(12)$ & $0.0253(12)$ & $0.0020(9)$ & $0.0015(9)$ & $0.0083(9)$ \\
C3 & $0.0235(12)$ & $0.0296(13)$ & $0.0199(11)$ & $0.0021(10)$ & $0.0022(10)$ & $0.0072(10)$ \\
C4 & $0.0209(11)$ & $0.0210(12)$ & $0.0236(12)$ & $0.0036(9)$ & $0.0026(9)$ & $-0.0015(10)$ \\
C5 & $0.0212(12)$ & $0.0160(12)$ & $0.0304(13)$ & $0.0036(9)$ & $0.0001(10)$ & $0.0049(10)$ \\
C6 & $0.0172(11)$ & $0.0211(12)$ & $0.0230(11)$ & $0.0008(9)$ & $-0.0021(9)$ & $0.0080(9)$ \\
C7 & $0.0152(10)$ & $0.0136(11)$ & $0.0215(11)$ & $0.0004(8)$ & $0.0032(9)$ & $0.0052(9)$ \\
C8 & $0.0222(11)$ & $0.0143(11)$ & $0.0216(11)$ & $0.0022(9)$ & $0.0025(9)$ & $0.0077(9)$ \\
C9 & $0.0198(11)$ & $0.0184(11)$ & $0.0179(11)$ & $0.0012(9)$ & $0.0031(9)$ & $0.0051(9)$ \\
C10 & $0.0143(10)$ & $0.0160(11)$ & $0.0195(11)$ & $0.0004(8)$ & $0.0028(9)$ & $0.0042(9)$ \\
C11 & $0.0239(12)$ & $0.0176(11)$ & $0.0198(11)$ & $0.0019(9)$ & $-0.0034(9)$ & $0.0054(9)$ \\
C12 & $0.0151(10)$ & $0.0142(11)$ & $0.0197(11)$ & $0.0000(8)$ & $-0.0022(9)$ & $0.0055(9)$ \\
C13 & $0.0148(10)$ & $0.0174(11)$ & $0.0160(10)$ & $0.0031(8)$ & $-0.0001(8)$ & $0.0040(9)$ \\
C14 & $0.0181(11)$ & $0.0154(11)$ & $0.0211(11)$ & $-0.0006(8)$ & $-0.0010(9)$ & $0.0066(9)$ \\
C15 & $0.0210(11)$ & $0.0141(11)$ & $0.0227(11)$ & $0.0027(9)$ & $-0.0009(9)$ & $0.0031(9)$ \\
C16 & $0.0206(11)$ & $0.0200(12)$ & $0.0216(11)$ & $0.0047(9)$ & $0.0048(9)$ & $0.0050(9)$ \\
C17 & $0.0171(11)$ & $0.0202(12)$ & $0.0239(11)$ & $-0.0005(9)$ & $0.0028(9)$ & $0.0090(9)$ \\
C18 & $0.0216(12)$ & $0.0363(14)$ & $0.0367(14)$ & $0.0030(10)$ & $0.0015(11)$ & $0.0153(12)$ \\
& & & & & &
\end{tabular}

Geometric parameters $\left(\AA,{ }^{\circ}\right)$

\begin{tabular}{llll}
\hline $\mathrm{O} 1-\mathrm{C} 2$ & $1.357(2)$ & $\mathrm{C} 7-\mathrm{C} 8$ & $1.385(3)$ \\
$\mathrm{O} 1-\mathrm{H} 1$ & 0.8400 & $\mathrm{C} 8-\mathrm{C} 9$ & $1.386(3)$ \\
$\mathrm{O} 2-\mathrm{C} 13$ & $1.362(2)$ & $\mathrm{C} 8-\mathrm{H} 8$ & 0.9500 \\
$\mathrm{O} 2-\mathrm{H} 2$ & 0.8400 & $\mathrm{C} 9-\mathrm{C} 10$ & $1.389(3)$ \\
$\mathrm{O} 3-\mathrm{C} 18$ & $1.416(3)$ & $\mathrm{C} 9-\mathrm{H} 9$ & 0.9500 \\
$\mathrm{O} 3-\mathrm{H} 3 \mathrm{~A}$ & 0.8400 & $\mathrm{C} 10-\mathrm{C} 12$ & $1.475(3)$
\end{tabular}




\begin{tabular}{|c|c|c|c|}
\hline $\mathrm{N} 1-\mathrm{N} 2$ & $1.286(2)$ & $\mathrm{C} 11-\mathrm{H} 11 \mathrm{~A}$ & 0.9800 \\
\hline $\mathrm{N} 1-\mathrm{C} 1$ & $1.410(2)$ & $\mathrm{C} 11-\mathrm{H} 11 \mathrm{~B}$ & 0.9800 \\
\hline $\mathrm{N} 2-\mathrm{C} 7$ & $1.371(2)$ & $\mathrm{C} 11-\mathrm{H} 11 \mathrm{C}$ & 0.9800 \\
\hline N3-C10 & $1.367(2)$ & $\mathrm{C} 12-\mathrm{C} 17$ & $1.393(3)$ \\
\hline $\mathrm{N} 3-\mathrm{C} 7$ & $1.380(3)$ & $\mathrm{C} 12-\mathrm{C} 13$ & $1.396(3)$ \\
\hline $\mathrm{N} 3-\mathrm{C} 11$ & $1.460(3)$ & $\mathrm{C} 13-\mathrm{C} 14$ & $1.388(3)$ \\
\hline $\mathrm{C} 1-\mathrm{C} 6$ & $1.397(3)$ & $\mathrm{C} 14-\mathrm{C} 15$ & $1.386(3)$ \\
\hline $\mathrm{C} 1-\mathrm{C} 2$ & $1.409(3)$ & $\mathrm{C} 14-\mathrm{H} 14$ & 0.9500 \\
\hline $\mathrm{C} 2-\mathrm{C} 3$ & $1.390(3)$ & $\mathrm{C} 15-\mathrm{C} 16$ & $1.382(3)$ \\
\hline $\mathrm{C} 3-\mathrm{C} 4$ & $1.375(3)$ & C15-H15 & 0.9500 \\
\hline $\mathrm{C} 3-\mathrm{H} 3$ & 0.9500 & $\mathrm{C} 16-\mathrm{C} 17$ & $1.378(3)$ \\
\hline $\mathrm{C} 4-\mathrm{C} 5$ & $1.389(3)$ & $\mathrm{C} 16-\mathrm{H} 16$ & 0.9500 \\
\hline $\mathrm{C} 4-\mathrm{H} 4$ & 0.9500 & C17-H17 & 0.9500 \\
\hline $\mathrm{C} 5-\mathrm{C} 6$ & $1.372(3)$ & C18-H18A & 0.9800 \\
\hline $\mathrm{C} 5-\mathrm{H} 5$ & 0.9500 & $\mathrm{C} 18-\mathrm{H} 18 \mathrm{~B}$ & 0.9800 \\
\hline $\mathrm{C} 6-\mathrm{H} 6$ & 0.9500 & $\mathrm{C} 18-\mathrm{H} 18 \mathrm{C}$ & 0.9800 \\
\hline $\mathrm{C} 2-\mathrm{O} 1-\mathrm{H} 1$ & 109.5 & $\mathrm{C} 10-\mathrm{C} 9-\mathrm{H} 9$ & 125.7 \\
\hline $\mathrm{C} 13-\mathrm{O} 2-\mathrm{H} 2$ & 109.5 & $\mathrm{~N} 3-\mathrm{C} 10-\mathrm{C} 9$ & $107.62(17)$ \\
\hline $\mathrm{C} 18-\mathrm{O} 3-\mathrm{H} 3 \mathrm{~A}$ & 109.5 & $\mathrm{~N} 3-\mathrm{C} 10-\mathrm{C} 12$ & $124.35(19)$ \\
\hline $\mathrm{N} 2-\mathrm{N} 1-\mathrm{C} 1$ & $113.64(18)$ & $\mathrm{C} 9-\mathrm{C} 10-\mathrm{C} 12$ & $128.03(18)$ \\
\hline $\mathrm{N} 1-\mathrm{N} 2-\mathrm{C} 7$ & $115.61(18)$ & $\mathrm{N} 3-\mathrm{C} 11-\mathrm{H} 11 \mathrm{~A}$ & 109.5 \\
\hline $\mathrm{C} 10-\mathrm{N} 3-\mathrm{C} 7$ & $108.49(17)$ & $\mathrm{N} 3-\mathrm{C} 11-\mathrm{H} 11 \mathrm{~B}$ & 109.5 \\
\hline $\mathrm{C} 10-\mathrm{N} 3-\mathrm{C} 11$ & $127.06(17)$ & $\mathrm{H} 11 \mathrm{~A}-\mathrm{C} 11-\mathrm{H} 11 \mathrm{~B}$ & 109.5 \\
\hline $\mathrm{C} 7-\mathrm{N} 3-\mathrm{C} 11$ & $124.41(17)$ & $\mathrm{N} 3-\mathrm{C} 11-\mathrm{H} 11 \mathrm{C}$ & 109.5 \\
\hline $\mathrm{C} 6-\mathrm{C} 1-\mathrm{C} 2$ & $118.50(19)$ & $\mathrm{H} 11 \mathrm{~A}-\mathrm{C} 11-\mathrm{H} 11 \mathrm{C}$ & 109.5 \\
\hline $\mathrm{C} 6-\mathrm{C} 1-\mathrm{N} 1$ & $115.80(19)$ & $\mathrm{H} 11 \mathrm{~B}-\mathrm{C} 11-\mathrm{H} 11 \mathrm{C}$ & 109.5 \\
\hline $\mathrm{C} 2-\mathrm{C} 1-\mathrm{N} 1$ & $125.66(19)$ & $\mathrm{C} 17-\mathrm{C} 12-\mathrm{C} 13$ & $118.08(19)$ \\
\hline $\mathrm{O} 1-\mathrm{C} 2-\mathrm{C} 3$ & $119.0(2)$ & $\mathrm{C} 17-\mathrm{C} 12-\mathrm{C} 10$ & 119.67 (19) \\
\hline $\mathrm{O} 1-\mathrm{C} 2-\mathrm{C} 1$ & $121.41(18)$ & $\mathrm{C} 13-\mathrm{C} 12-\mathrm{C} 10$ & $122.00(19)$ \\
\hline $\mathrm{C} 3-\mathrm{C} 2-\mathrm{C} 1$ & $119.6(2)$ & $\mathrm{O} 2-\mathrm{C} 13-\mathrm{C} 14$ & $121.74(19)$ \\
\hline $\mathrm{C} 4-\mathrm{C} 3-\mathrm{C} 2$ & $120.4(2)$ & $\mathrm{O} 2-\mathrm{C} 13-\mathrm{C} 12$ & $117.65(18)$ \\
\hline $\mathrm{C} 4-\mathrm{C} 3-\mathrm{H} 3$ & 119.8 & $\mathrm{C} 14-\mathrm{C} 13-\mathrm{C} 12$ & $120.60(19)$ \\
\hline $\mathrm{C} 2-\mathrm{C} 3-\mathrm{H} 3$ & 119.8 & $\mathrm{C} 15-\mathrm{C} 14-\mathrm{C} 13$ & $119.8(2)$ \\
\hline $\mathrm{C} 3-\mathrm{C} 4-\mathrm{C} 5$ & $120.6(2)$ & $\mathrm{C} 15-\mathrm{C} 14-\mathrm{H} 14$ & 120.1 \\
\hline $\mathrm{C} 3-\mathrm{C} 4-\mathrm{H} 4$ & 119.7 & $\mathrm{C} 13-\mathrm{C} 14-\mathrm{H} 14$ & 120.1 \\
\hline $\mathrm{C} 5-\mathrm{C} 4-\mathrm{H} 4$ & 119.7 & $\mathrm{C} 16-\mathrm{C} 15-\mathrm{C} 14$ & $120.3(2)$ \\
\hline $\mathrm{C} 6-\mathrm{C} 5-\mathrm{C} 4$ & $119.3(2)$ & $\mathrm{C} 16-\mathrm{C} 15-\mathrm{H} 15$ & 119.9 \\
\hline $\mathrm{C} 6-\mathrm{C} 5-\mathrm{H} 5$ & 120.3 & $\mathrm{C} 14-\mathrm{C} 15-\mathrm{H} 15$ & 119.9 \\
\hline $\mathrm{C} 4-\mathrm{C} 5-\mathrm{H} 5$ & 120.3 & $\mathrm{C} 17-\mathrm{C} 16-\mathrm{C} 15$ & $119.5(2)$ \\
\hline $\mathrm{C} 5-\mathrm{C} 6-\mathrm{C} 1$ & $121.5(2)$ & $\mathrm{C} 17-\mathrm{C} 16-\mathrm{H} 16$ & 120.3 \\
\hline $\mathrm{C} 5-\mathrm{C} 6-\mathrm{H} 6$ & 119.3 & $\mathrm{C} 15-\mathrm{C} 16-\mathrm{H} 16$ & 120.3 \\
\hline $\mathrm{C} 1-\mathrm{C} 6-\mathrm{H} 6$ & 119.3 & $\mathrm{C} 16-\mathrm{C} 17-\mathrm{C} 12$ & $121.7(2)$ \\
\hline $\mathrm{N} 2-\mathrm{C} 7-\mathrm{N} 3$ & $117.55(18)$ & $\mathrm{C} 16-\mathrm{C} 17-\mathrm{H} 17$ & 119.2 \\
\hline $\mathrm{N} 2-\mathrm{C} 7-\mathrm{C} 8$ & $133.93(19)$ & $\mathrm{C} 12-\mathrm{C} 17-\mathrm{H} 17$ & 119.2 \\
\hline $\mathrm{N} 3-\mathrm{C} 7-\mathrm{C} 8$ & $108.49(17)$ & $\mathrm{O} 3-\mathrm{C} 18-\mathrm{H} 18 \mathrm{~A}$ & 109.5 \\
\hline $\mathrm{C} 7-\mathrm{C} 8-\mathrm{C} 9$ & $106.85(19)$ & $\mathrm{O} 3-\mathrm{C} 18-\mathrm{H} 18 \mathrm{~B}$ & 109.5 \\
\hline $\mathrm{C} 7-\mathrm{C} 8-\mathrm{H} 8$ & 126.6 & $\mathrm{H} 18 \mathrm{~A}-\mathrm{C} 18-\mathrm{H} 18 \mathrm{~B}$ & 109.5 \\
\hline
\end{tabular}




$\begin{array}{llll}\mathrm{C} 9-\mathrm{C} 8-\mathrm{H} 8 & 126.6 & \mathrm{O} 3-\mathrm{C} 18-\mathrm{H} 18 \mathrm{C} & 109.5 \\ \mathrm{C} 8-\mathrm{C} 9-\mathrm{C} 10 & 108.55(18) & \mathrm{H} 18 \mathrm{~A}-\mathrm{C} 18-\mathrm{H} 18 \mathrm{C} & 109.5 \\ \mathrm{C} 8-\mathrm{C} 9-\mathrm{H} 9 & 125.7 & \mathrm{H} 18 \mathrm{~B}-\mathrm{C} 18-\mathrm{H} 18 \mathrm{C} & 109.5 \\ \mathrm{C} 1-\mathrm{N} 1-\mathrm{N} 2-\mathrm{C} 7 & -177.49(17) & \mathrm{C} 7-\mathrm{C} 8-\mathrm{C} 9-\mathrm{C} 10 & -0.8(2) \\ \mathrm{N} 2-\mathrm{N} 1-\mathrm{C} 1-\mathrm{C} 6 & -179.85(17) & \mathrm{C} 7-\mathrm{N} 3-\mathrm{C} 10-\mathrm{C} 9 & -0.6(2) \\ \mathrm{N} 2-\mathrm{N} 1-\mathrm{C} 1-\mathrm{C} 2 & 2.6(3) & \mathrm{C} 11-\mathrm{N} 3-\mathrm{C} 10-\mathrm{C} 9 & 177.13(18) \\ \mathrm{C} 6-\mathrm{C} 1-\mathrm{C} 2-\mathrm{O} 1 & 179.88(19) & \mathrm{C} 7-\mathrm{N} 3-\mathrm{C} 10-\mathrm{C} 12 & 178.51(18) \\ \mathrm{N} 1-\mathrm{C} 1-\mathrm{C} 2-\mathrm{O} 1 & -2.6(3) & \mathrm{C} 11-\mathrm{N} 3-\mathrm{C} 10-\mathrm{C} 12 & -3.7(3) \\ \mathrm{C} 6-\mathrm{C} 1-\mathrm{C} 2-\mathrm{C} 3 & -0.9(3) & \mathrm{C} 8-\mathrm{C} 9-\mathrm{C} 10-\mathrm{N} 3 & 0.9(2) \\ \mathrm{N} 1-\mathrm{C} 1-\mathrm{C} 2-\mathrm{C} 3 & 176.63(19) & \mathrm{C} 8-\mathrm{C} 9-\mathrm{C} 10-\mathrm{C} 12 & -178.2(2) \\ \mathrm{O} 1-\mathrm{C} 2-\mathrm{C} 3-\mathrm{C} 4 & 179.32(19) & \mathrm{N} 3-\mathrm{C} 10-\mathrm{C} 12-\mathrm{C} 17 & 128.7(2) \\ \mathrm{C} 1-\mathrm{C} 2-\mathrm{C} 3-\mathrm{C} 4 & 0.1(3) & \mathrm{C} 9-\mathrm{C} 10-\mathrm{C} 12-\mathrm{C} 17 & -52.3(3) \\ \mathrm{C} 2-\mathrm{C} 3-\mathrm{C} 4-\mathrm{C} 5 & 1.1(3) & \mathrm{N} 3-\mathrm{C} 10-\mathrm{C} 12-\mathrm{C} 13 & -57.2(3) \\ \mathrm{C} 3-\mathrm{C} 4-\mathrm{C} 5-\mathrm{C} 6 & -1.4(3) & \mathrm{C} 9-\mathrm{C} 10-\mathrm{C} 12-\mathrm{C} 13 & 121.8(2) \\ \mathrm{C} 4-\mathrm{C} 5-\mathrm{C} 6-\mathrm{C} 1 & 0.6(3) & \mathrm{C} 17-\mathrm{C} 12-\mathrm{C} 13-\mathrm{O} 2 & 176.58(17) \\ \mathrm{C} 2-\mathrm{C} 1-\mathrm{C} 6-\mathrm{C} 5 & 0.6(3) & \mathrm{C} 10-\mathrm{C} 12-\mathrm{C} 13-\mathrm{O} 2 & 2.4(3) \\ \mathrm{N} 1-\mathrm{C} 1-\mathrm{C} 6-\mathrm{C} 5 & -177.19(18) & \mathrm{C} 17-\mathrm{C} 12-\mathrm{C} 13-\mathrm{C} 14 & -2.3(3) \\ \mathrm{N} 1-\mathrm{N} 2-\mathrm{C} 7-\mathrm{N} 3 & 179.37(17) & \mathrm{C} 10-\mathrm{C} 12-\mathrm{C} 13-\mathrm{C} 14 & -176.47(18) \\ \mathrm{N} 1-\mathrm{N} 2-\mathrm{C} 7-\mathrm{C} 8 & 1.5(3) & \mathrm{O} 2-\mathrm{C} 13-\mathrm{C} 14-\mathrm{C} 15 & -177.29(17) \\ \mathrm{C} 10-\mathrm{N} 3-\mathrm{C} 7-\mathrm{N} 2 & -178.23(17) & \mathrm{C} 12-\mathrm{C} 13-\mathrm{C} 14-\mathrm{C} 15 & 1.5(3) \\ \mathrm{C} 11-\mathrm{N} 3-\mathrm{C} 7-\mathrm{N} 2 & 3.9(3) & \mathrm{C} 13-\mathrm{C} 14-\mathrm{C} 15-\mathrm{C} 16 & 0.0(3) \\ \mathrm{C} 10-\mathrm{N} 3-\mathrm{C} 7-\mathrm{C} 8 & 0.2(2) & \mathrm{C} 14-\mathrm{C} 15-\mathrm{C} 16-\mathrm{C} 17 & -0.8(3) \\ \mathrm{C} 11-\mathrm{N} 3-\mathrm{C} 7-\mathrm{C} 8 & -177.69(18) & \mathrm{C} 15-\mathrm{C} 16-\mathrm{C} 17-\mathrm{C} 12 & 0.0(3) \\ \mathrm{N} 2-\mathrm{C} 7-\mathrm{C} 8-\mathrm{C} 9 & 178.4(2) & \mathrm{C} 13-\mathrm{C} 12-\mathrm{C} 17-\mathrm{C} 16 & 1.5(3) \\ \mathrm{N} 3-\mathrm{C} 7-\mathrm{C} 8-\mathrm{C} 9 & 0.4(2) & \mathrm{C} 10-\mathrm{C} 12-\mathrm{C} 17-\mathrm{C} 16 & 175.89(18) \\ & & & \end{array}$

Hydrogen-bond geometry $\left(A,{ }^{\circ}\right)$

$\mathrm{Cg} 1$ and $\mathrm{Cg} 2$ are the centroids of the $\mathrm{C} 1-\mathrm{C} 6$ and $\mathrm{C} 12-\mathrm{C} 17$ rings, respectively

\begin{tabular}{lllll}
\hline$D-\mathrm{H} \cdots A$ & $D-\mathrm{H}$ & $\mathrm{H} \cdots A$ & $D \cdots A$ & $D-\mathrm{H} \cdots A$ \\
\hline $\mathrm{O} 1-\mathrm{H} 1 \cdots \mathrm{N} 2$ & 0.84 & 1.81 & $2.530(2)$ & 143 \\
$\mathrm{O} 2-\mathrm{H} 2 \cdots \mathrm{O} 3^{\mathrm{i}}$ & 0.84 & 1.81 & $2.641(2)$ & 171 \\
$\mathrm{O} 3-\mathrm{H} 3 A \cdots \mathrm{O} 1^{\mathrm{ii}}$ & 0.84 & 1.97 & $2.763(2)$ & 157 \\
$\mathrm{C} 11-\mathrm{H} 11 B \cdots C g 1^{\mathrm{iii}}$ & 0.98 & 2.73 & $3.587(2)$ & 147 \\
$\mathrm{C} 18-\mathrm{H} 18 C \cdots C g 2^{\text {iv }}$ & 0.98 & 2.75 & $3.483(3)$ & 132 \\
\hline
\end{tabular}

Symmetry codes: (i) $-x+1,-y+2,-z+1$; (ii) $x, y, z+1$; (iii) $-x,-y+1,-z$; (iv) $-x,-y+2,-z+1$. 